\title{
Application of Value Engineering in Cost Management of Construction Engineering
}

\section{Pengfei Feng}

Huatong Real Estate Co., Ltd., Beijing, 100000, China

\begin{abstract}
Value engineering is actually through a series of technical methods to improve the value of construction engineering, and to ensure the effective use of various resources management work. In the current development of construction engineering, value engineering has been widely used in various an important technology for enterprises to effectively allocate resources, reduce construction costs, and improve the quality of financial management. In this context, this paper focuses on the analysis of the application of value engineering in construction engineering cost management, and discusses specific application countermeasures based on the principles of value engineering, hoping to provide references for relevant personnel.
\end{abstract}

\section{Keywords}

value engineering; construction project; cost management; application

\section{价值工程在建筑工程成本管理方面的应用}

\section{冯鹏飞}

华通置业有限公司, 中国・北京 100000

\section{摘 要}

价值工程实际上就是通过一系列的技术方法来提高建筑工程的价值，并且确保各项资源有效利用的管理工作。在目前的建筑 工程发展中, 价值工程已经有非常广泛的应用, 是各企业有效调配资源、缩短施工成本、提高财务管理质量的重要技术。介 于此, 论文着重于对价值工程在建筑工程成本管理工作中的应用进行分析, 以价值工程原理出发来探讨具体的应用对策, 希 望能够为相关人员提供参考。

\section{关键词}

价值工程；施工项目；成本管理；应用

\section{1 引言}

施工项目成本是各施工企业必须考虑的问题, 而在建筑 工程体系中, 加强对施工项目成本管理质量是非常重要的内 容。就目前来看, 影响到施工项目成本的主要有两方面内容: 第一, 是在市场发展中因为市场经济的问题而导致施工项目 成本受到影响; 第二, 则是因为施工工程及其相关企业自身 经营方面的相应影响因素。

可以说, 建筑工程中项目成本包括整体工程的所有费用, 所以要想更好地提高经济效益, 就需要建筑企业能够充分地 把握好整体成本, 通过价值工程来降低成本, 进而达到较好

【作者简介】冯鹏飞(1986-), 男, 中国河北保定人, 本科学历, 造价工程师, 从事成本管理研究。
的经济效益。

\section{2 价值工程的相关概述}

\section{1 价值工程原理}

在项目成本的管理工作中, 为了能够更好地降低成本并 保证施工质量，施工单位一般都需要对各项支出和资源的应 用进行有效的分析，并制定相应的使用策略，进而达到对资 源的有效调配和成本的降低, 而该工作则是价值工程的原型 所在。随着时代的发展, 实际上价值工程已经发展成为一项 以信息技术和现代化管理理念为基础的科学。

\section{在现阶段的建筑工程中, 财务管理人员将对工程的具体} 职能进行分析, 明确各项目的成本支出项目, 使得产品能够 更好地提升其使用价值, 通过最低的成本来实现建筑工程的 
目标。在建筑工程的开展中, 业主并不只是为了建筑体本身 而购买建筑产品, 其更多的是要获取建筑工程的各项功能和 服务, 所以现阶段价值工程也在朝着建筑体以外的相关内容 来进行控制，控制功能过剩和不足的问题 ${ }^{[1]}$ 。

\section{2 加强价值工程对施工项目成本管理的对策}

要想更好地提高成本管理质量, 施工单位必须加强价 值工程的应用, 论文针对这些问题提出几点价值工程的应用 对策。

\subsection{1 对施工工程的整体项目进行严格的分析和研究}

为了能够更好地明确在建筑工程体系中各项目的支出和 相应的经济活动, 在整体的施工活动中, 财务管理人员必须 对项目的具体开展情况, 结合施工项目的设计方案和预算来 确定各项目价值。实际上, 为了能够更好地提高价值工程的 质量, 还需要财务管理人员能够针对在建筑工程各项目中使 用的材料、技术、施工方法等进行严格的分析, 并研究这些 因素的施工可行性和经济性, 从这一点为突破点来控制实际 建筑工程的成本, 减少建筑工程的成本支出, 进而更好的在 保证建筑成品价值的条件下减少生产的成本 ${ }^{[2]}$ 。

\subsection{2 保证产品质量并同时减少各方面的支出}

在建筑工程项目的开展过程中, 施工单位一般都需要 通过各项管理办法来提高建筑成品的质量和施工的质量, 对 整体的施工项目进行严格的把握, 只有在确保整体建筑工程 施工质量的前提下才能够更好地开展价值工程, 减少一些不 必要的支出情况。财务人员必须明确一点, 那就是要严格避 免技术的提升和成本降低两者因素的分离, 实际上这两者是 可以共存的，在保证资源的有效应用和技术的应用质量下可 以有效地降低施工的成本, 把握好建筑产品的质量和成本的 关系。

\subsection{3 提高管理人员的素养和管理技术的应用}

在对整体建筑工程项目进行管理时, 施工企业必须加强 对整体建筑工程的管理, 其中特别是对施工人员和管理人员 的管理。因为管理工作极大地影响到整体施工的质量, 同时 还影响到各项建筑资源和技术资源的有效应用, 这就牵涉到 了关于财务管理的问题。所以, 为了能够更好地提高价值工 程的质量, 施工单位必须加强对管理人员的管理, 要确保管 理人员能够具备扎实的管理能力和经验, 在各部门的通力合 作之下来对整个生产过程进行管理, 进而更好的加强成本的
管理效果。

\section{3 价值工程的内容}

\section{1 功能、价值和成本的关系}

功能、价值以及成本三个因素是建筑工程中三个具有紧 密联系且十分重要的内在因素。具体来说, 价值是功能与费 用的商, 这也就明确表示了工程的总体成本支出不变的条件 下, 产品的功能和价值是成正比的关系。对于建筑工程来说, 其服务质量越好、产品性能越强，那么其价值也就越大。所 以基于这个原理, 在价值工程的开展过程中为了能够更好地 提高管理质量, 可以采取以下几点措施。

第一, 要尽量降低产品的生产成本, 这一点需要注意的 是必须在保障产品的功能不变的情况下进行。

第二, 要在提升建筑工程的功能的过程中尽量地降低产 品的生产成本。

第三, 在保障产品成本不变的情况下尽量提高产品的 功能。

第四, 在稍微提高产品成本的条件下大幅度提升产品的 功能。

第五, 略微减少产品的功能, 使得其能够大幅减少在生 产上投入的费用。

根据这几点可以看出, 在开展价值工程的过程中必须做 到全方位的考虑, 进行综合的分析来进行施工的成本管理 ${ }^{[3]}$ 。

\section{2 通过价值工程来进行成本控制工作}

在工程的施工过程中, 施工单位需要特别对各个项目进 行整体的考虑, 要从工程的设计方案出发, 发现设计方案是 否能够满足工程的具体需求, 并且对方案之中的技术体系和 施工工艺进行分析, 明确其存在的具体缺陷, 特别是经济上 的缺陷。同时, 需要加强对预算体系和项目管理的改良, 减 少成本。就目前来看, 要想更好地提高价值工程的质量, 在 现阶段的建筑工程中有两方面的方法。一是对各类施工方案 进行全面的分析研究, 并研究和分析最好的施工方案; 二是 对已经设计好的施工方案进行改进，进行进一步的改善。论 文则是针对第二点进行着重分析:

首先, 为了能够更好地提高价值工程质量, 需要确定好 对优化对象的确定。优化对象主要是指的是对整体建筑工程 的施工方案、技术体系、预算管理等进行研究分析, 研究在 这之中是否存在改进空间, 从而得到更好的施工方案。 
其次, 要根据建筑体系的设计图纸和方案来对建筑体系 的各项功能进行分析, 特别是要研究建筑工程的具体作用, 选择合适的功能, 对这些功能进行合理的删增, 去除一些不 必要的功能。同时, 管理人员需要加强对产品功能的设计, 计算各个部分占的比重。一般来说, 在开展该工作的过程中, 相关人员需要重视两个方面。一是确定好各功能的重点与比 重, 要分配好施工资源, 计算功能系数, 对各个功能比重因 子进行对比; 二是要明确自身所具有的资源体系, 方便后续 调配。

最后, 要确定好改进对象和对产品的成本系数和价值系 数进行计算。对于前者来说, 施工团队需要定时对工程所存 在的问题进行协商与分析，找到具体的问题并加以解决; 对 于后者来说则是需要做好计算工作。在成本系数的计算中是 将分部工程预算成本与总成本相处, 并通过分部工程功能系 数与分部工程成本系数相除来获得价值系数 ${ }^{[4]}$ 。

\section{4 结语}

综上所述，论文探讨了价值工程在建筑工程体系成本管 理工作中的具体应用。可以说成本管理是目前建筑工程管理 中非常重要的内容, 这关乎到建筑工程的经济效益, 通过价 值工程可以有效提高成本管理质量, 这是当前各建筑企业需 要着重研究的内容。

\section{参考文献}

[1] 胡水清. 快乐体育在中学体育教育中的应用研究 [J]. 体育科技文 献通报 ,2018,26(11):104-107

[2] 罗永义, 仇军. 基于体育本体论的 “快乐体育” 教学理念反思 [J] 北京体育大学学报, 2015,38(02):105-111.

[3] 祁龙祥, 焚晨. 快乐体育在中学篮球体育教学中应用的研究 [J]. 南 京体育学院学报 (社会科学版),2010,24(06):109-111.

[4] 王文山. 快乐体育在中学体育教学中应用的若干思考 [J]. 哈尔滨 体育学院学报, 2007(04):87-89. 